\title{
Programm- Und Organisationskomitee
}

Gemeinsame Jahrestagung der Deutschen und der Österreichischen Gesellschaft für Hämatologie und Onkologie

Hamburg, 8.-11. Oktober 1995

PROGRAMM- UND ORGANISATIONSKOMITEE

Kongreßpräsident:

Prof. Dr. D. K. Hossfeld, Hamburg

Kongreßsekretär:

PD Dr. U. Dührsen, Hamburg

Lokales Organisationskomitee:

Prof. Dr. D.K. Hossfeld, Hamburg Dr. H. Erdmann, Hamburg Prof. Dr. W. Ostertag, Hamburg

Prof. Dr. K. Winkler, Hamburg

Wissenschaftliches Komitee:

Prof. Dr. W.E. Berdel, Berlin

Prof. Dr. V. Diehl, Köln

Prof. Dr. E. Heidemann, Stuttgart

Prof. Dr. W. Hiddemann, Göttingen

Prof. Dr. Ch. Huber, Mainz

Prof. Dr. W. Knapp, Wien

Prof. Dr. H. Ludwig, Wien

Prof. Dr. M. Pfreundschuh, HomburE

Prof. Dr. U.W. Schaefer, Essen

PD Dr. U. Dührsen, Hamburg Prof. Dr. R. Kuse, Hamburg Prof. Dr. H.-J. Weh, Hamburg Prof.

Dr. A.R. Zander, Hamburg

Prof. Dr. G. Brittinger, Essen

Prof. Dr. M. Freund, Rostock

Doz. Dr. R. Heinz, Wien

Prof. Dr. K. Höffken, Jena

Prof. Dr. D. Huhn, Berlin

Prof. Dr. K. Lechner, Wien

Prof. Dr. D. Niederwieser, Innsbruck

Prof. Dr. W. Queißer, Mannheim

Prof. Dr. S. Seeber, Essen

Organisation der Fortbildung für Schwestern und Pfleger:

Schwester A. Hussel, Essen 